\title{
Stress analysis of implant-supported removable partial denture with anterior fixed prostheses and conventional implant supported overdentures in the edentulous mandible
}

\section{Gulsum Sayin Ozel ( $\square$ gozel@medipol.edu.tr)}

Istanbul Medipol University https://orcid.org/0000-0001-8833-5259

Ozgur Inan

Selcuk University

Ozgun Yusuf Ozyilmaz

Bezmialem Vakif University

\section{Research article}

Keywords: Dental implants, Implant-supported removable dentures, Stress transmission

Posted Date: April 10th, 2020

DOI: https://doi.org/10.21203/rs.2.12325/v2

License: (c) (i) This work is licensed under a Creative Commons Attribution 4.0 International License.

Read Full License 


\section{Abstract}

Background: Recently, a new alternative treatment protocols without complex surgical or grafting procedures has become popular in clinical dentistry. A new treatment concept is implant-supported removable partial dentures retained with anterior fixed prosthesis in edentulous mandible. The purpose of this study was to compare this new design implant supported removable partial dentures, with a conventional locater and bar attached implant overdenture prostheses retained by two or four implants via photoelastic stress analysis.

Methods: This study was designed to evaluate the force transmission of commercially available titanium implants inserted into edentulous mandibles composed of photoelastic resin within two to four implants using two different types of prosthesis: overdentures and implant-retained removable partial dentures. In all models, implants were placed parallel to each other and the midline. A $300 \mathrm{~N}$ load was applied to the first premolars. Loading and stress distributions were photographed after the completion of the prostheses.

Results: In the models with two implant-retained removable partial dentures, the stress distribution was found to be lower than that with the four implant-retained removable partial dentures. The locator, a stud attachment, showed the most equitable stress distribution for overdenture models. Despite this, nonsplinted implants caused high stresses around the distal implant on the loading side.

Conclusions: When models were compared, the stress loads were transmitted to other implants by splinting. Implant-supported removable partial dentures with an anterior fixed prosthetic design may serve as an acceptable and affordable alternative treatment option. These dentures appear to be advantageous in terms of stress transmission considering effects such as ease of use, esthetic requirements, patient requests, and reduction in the area covered by the prosthesis.

\section{Background}

The use of a minimal number of implants, easier surgical procedures, and simpler prosthetic designs with prefabricated attachments are the most commonly known advantages of mandibular implant-retained overdentures. ${ }^{1,2}$ The number and location of implants are important factors for stress distribution around implants and the supporting bone. ${ }^{3,4}$ Besides, the type of the attachment is also an important factor for implant-supported overdentures (IO). Unsplinted attachment as locater or splinted attachment as bar can be used for 10 according to retention necessity ${ }^{3}$. In addition to retention capacity, stress transmission between the residual ridges of the implants, implant angulation, and patient satisfaction with both esthetic and functional properties are very important for choosing the correct attachment type and prosthetic design ${ }^{2}$. Previous studies; focused on the selection the type of attachment system, it is important to consider that the system must allow for optimum stress distribution around the implants, provide the most equal load transmission (both for soft tissue and the implant complex), and protect the 
bone from stress within physiological limits. This way, undesirable stress and complications are avoided, and bone may be preserved. ${ }^{5,6}$

In recent years, a new alternative treatment protocol without complex surgical or grafting procedures has become popular in clinical dentistry. This new protocol is implant- supported fixed prosthesis into the interforaminal region with bilateral movable partial dentures. ${ }^{7}$ This concept can provide a fixed restoration in the esthetic region that is not necessary to remove at night, has minimal or smaller removable prosthesis components, and easily compensates implant angulation. For the edentulous mandible, as well as 10 prosthesis and fixed implant-supported prosthesis, an implant-supported fixed prosthesis with two or four implants in the anterior region combined with removable partial dentures may comprise a new alternative treatment protocol. ${ }^{8}$ There are many clinical reports of this new treatment method in the literature, ${ }^{7-11}$ but there is no comparison or evaluation of the stress distribution.

Therefore, the aim of this in-vitro study was to compare this new anterior implant-supported fixed prosthesis combination with removable partial dentures, with a conventional locater and bar attached implant overdenture prosthesis on two and four implant-supported photoelastic edentulous models, to evaluate the ideal attachment type for this new design prosthesis and to examine the stress transmission of this increasingly popular treatment protocol on periimplant areas. The null hypothesis was that there is no significant difference in stress transmission between different prosthetic designs.

\section{Methods}

This study was performed in Selcuk University, Faculty of Dentistry Research Laboratory.

For the two-implant edentulous model, four different types of prosthesis;

- overdenture with locator attachment,

- crown design retained removable prosthesis with clasp retention,

- bridge design retained removable prosthesis with clasp retention,

- bridge design retained removable prosthesis with precision attachment retention,

and for the four-implant edentulous model, three different types of prosthesis

- prosthesis with bar attachment overdenture,

- fixed bridge design retained removable prosthesis with clasp retention,

- fixed retained removable prosthesis with precision attachment retention

were compared for stress distribution in a photoelastic stress analysis. 


\section{Preparation of acrylic and photoelastic models}

Six heat polymerized acrylic resin models (Kulzer Dental) were prepared to model an edentulous patient's mandible $(200 \mathrm{~mm} * 100 \mathrm{~mm} * 30 \mathrm{~mm})$. Cylindrical implants $(4.1 \times 12 \mathrm{~mm}$ tissue level, Straumann, Institut Straumann AG) were embedded in the parasymphyseal area of each wax model by a parallelometer (Orthofex, Fogászat Gyártơ KFT). The implants were placed parallel to each other and the midline. For the two-implant models, the distance between the implants were set to $22 \mathrm{~mm}$ and the implants were located in the lateral-canine region. ${ }^{4}$ For the four-implant models, the distance was set to $11 \mathrm{~mm}$. The acrylic models with implants were duplicated with a $\mathrm{C}$ type silicone impression material by the puttywash technique (Zetaplus, Zhermack). Silicone molds were cured for 1 week at $25^{\circ} \mathrm{C}$ to eliminate the tensions that can occur in photoelastic models depending on the shrinkage of the silicone. The curing was performed to avoid the inaccurate and redundant fringes arising from polymerization shrinkage. The models were tested for residual stresses after setting and after the curing period of 1 week. The Photoelastic resin (PL-2; $35 \mathrm{~g}$ ) and the catalyzer (PLH-2; $35 \mathrm{~g}$ ) (Measurements Group Inc.) were used. Manufacturer recommended formula was used to calculate the ratio of resin.

Both resin and silicone molds were placed into an incubator for 1 hour and heated to $46-52^{\circ} \mathrm{C}$. This heating process provided the lower viscosity and produced a more homogeneous mixture. After incubation, the catalyzer and resin were mixed with a glass pipette avoiding excessive movements and bubbles. The mixing resin filled the entire silicone molds. Mixtures in the silicone molds were polymerized at $25^{\circ} \mathrm{C}$ for 24 hours. Following this, all resin models were prepared and polished, and the prosthetic laboratory processes were started. For all prosthetic designs, silicone molds were used for standardization of settings for all steps like wax modelation, porcelain application, and artificial teeth settings. For light transmission, transparent acrylic resin (Akribel Hot Acryl, Atlas Enta Dental) was used according to the manufacturer's recommendations ${ }^{12}$ for all types of prosthesis. For gingival stimulation, the posterior areas of the prosthesis were removed at $3 \mathrm{~mm}$ thickness using a drill that marked $3 \mathrm{~mm}$ in advance to guide the removal. After removal of the prosthesis, the areas were filled with an artificial gingival mask (Zhermack Gingifast Elastic) and placed on the photoelastic models, and the outflows were removed for all types of prosthesis.

\section{Preparation of the implant-supported removable partial dentures}

For the fixed components of implant-supported removable partial dentures (IRPD), $5.5 \mathrm{~mm}$ solid abutments were placed into the two- and four-implant models and torqued with $35 \mathrm{Ncm}$. For the laboratory stages, laboratory models were duplicated from the photoelastic models with vinylpolysiloxane impression material (Virtual, Ivoclar Vivadent). First, two and four implant-supported bridge design restorations were modeled, and then a silicone index was used to standardize the bridge 
precision attachment and crown design models. An extracoronal resilient precision attachment was used (Probolte Attachments, Kargı Dental). With small dimensions (2.0 mm height and $4.2 \mathrm{~mm}$ length), these attachments are used particularly in anterior regions with two abutment teeth. Good retention, lack of milling requirements, and simplicity of use are the most important properties of this system. ${ }^{8}$ The casting procedures were performed according to the manufacturers' recommendations with a $\mathrm{Ni}-\mathrm{Cr}$ alloy $(61 \% \mathrm{Ni}$, $25.60 \% \mathrm{Cr}, 10.94 \% \mathrm{Mo}, 1.51 \% \mathrm{SI}, 0.01 \% \mathrm{Mn}$, and $0.01 \% \mathrm{C}$ ).

After the laying procedure, metals were sandblasted with 25-70 $\mu \mathrm{m} \mathrm{Al}_{2} \mathrm{O}_{3}$ particles (Star Dental) and ultrasonically cleaned for 15 minutes before porcelain application. Porcelain (Shofu Vintage Halo, Shofu Inc., PN 6507) was applied and then checked on the master models.

For the removable prosthesis with clasps, I bar clasps were planned. All models were cast with a Cr-Co alloy; 64\% Co, 28.5\% Cr, 8\% Fe, and 3\% Mo (Wironium Plus, Bego, PN 50190) After the artificial teeth were set for all models. After checking the setting on the master models, the finishing procedures were made with standard methods. Laying and polishing procedures were then conducted.

\section{Preparation of the implant-supported overdentures}

Initially, for the four IO models, SynOcta abutments (Straumann, Institut Straumann AG) and gold copings were screwed on. Bar attachments were cut according to the distance between the copings and blocked out with utility wax in order to set them $5 \mathrm{~mm}$ away from the tissue $(3 \mathrm{~mm}$ gingival height, $2 \mathrm{~mm}$ distance from the tissue). The prepared bars were adjusted by millimeters, a pattern resin was used for splitting the bars, and the gold copings were unscrewed for the soldering procedures.

For the two-implant models, locater abutments were fitted and torqued with a $35 \mathrm{~N} / \mathrm{cm}$ force, and an acrylic base plaque was prepared and fixed to the locater matrix. After the positioning of the attachments, models were duplicated for the laboratory procedures. Artificial teeth settings were made according to the silicone indexes. All finishing procedures were completed according to the standard methods on the duplicate models. After the laying and polishing procedures, the prostheses were checked on the master models. A blue-colored retentive rubber with a retention force of $1.5 \mathrm{lbs}=66.7 \mathrm{~N}$ was used.

A $300 \mathrm{~N}$ vertical load was applied bilaterally to the central fossa of the first premolar occlusal surface by a universal test machine (TSTM 02500, Elista Ltd.). The models were placed a special clamp-like device for stabilization so that there was no movement when the load was applied. During installation, the resulting stresses -were seen as the stress lines (Fringes)- were observed and recorded photographically (Powershot G3, Canon) in the field of a circular polariscope (Sharples 2026). The polariscope was placed into the universal test machine; the placement order from back to front was as follows: white light, source polaroid plates, the model, quarter-wave plates, and the photographic machine (Fig. 1). 
The stress fringes showed some properties such as color, frequency, and distribution that state the amount of strain. Fringes were composed of different color bands (isochromatic), and the border of these isochromatic fringes was determined by the amount of strain. The characteristic properties of isochromatic fringes are expressed numerically in Table $1 .{ }^{13,14}$

\section{Results}

Loading on the right and left side produced similar fringe patterns. Therefore, only results from the right side are presented. In particular, medium level stresses occurred in the regions numbered 1, 2, 3, and 4 (1.08-1.39 N) in the region of the implant on the loading side for 10 prostheses with locator attachment that were combined implant-tissue supported. In addition, the fringes occurred in the crest region on the loading side. Low levels of stress generation were observed in the regions numbered 6 and $10(0.28 \mathrm{~N})$. The stress values in the apical regions of the implants were higher than in the neck regions (Fig. 2a).

Moderate levels of stress generation in region $8(1.63 \mathrm{~N})$ for the IRPD with bridge design and clasp attachment. More fringes were occured in the apical than the neck region of the implants. A higher level of stress accumulation was observed on the loading side in the neck region of the implant regions 1-5 $(1.00 \mathrm{~N})$ than that in the neck region of the opposite side implant regions 6-10 (0.45-0.28 N) (Fig. 2b). Stress generation in region 8 for the two IRPD bridge+precision attachment was $1.08 \mathrm{~N}$. Low levels of fringe line formation in regions $1,5,6$, and $10(0.28 \mathrm{~N})$ was observed, and that the stress accumulation in the neck regions was lower in the models with precision attachment (Fig. 2c). Stress generation in region 8 of the IRPD with a crown design and clasp attachment was $2.00 \mathrm{~N}$ and in regions 1 was $1.22 \mathrm{~N}$ and in region 5 was $1.00 \mathrm{~N}$. The stress concentration was higher in the apical regions of the implants than in the cervical regions of the implants. (Fig. 2d, Fig. 3).

A greater concentration of stress was observed in the overdenture prosthesis with bar attachment IO than the locator attachment. However, stress concentrations occurring not only on the loading side implants but also on the opposite side implants (regions 11, 13, 15, and 18; 1.22-1.08 N. Fringes formed with reduced tissue support were less sparse than those in the edentulous areas. (Fig. 4a). Medium level stress (1.08-1.39 N) fringes occurring in regions $1,3,5,6,8,11,13,16$, and 18 for the IRPD with clasp attachments. Lower stress concentrations were observed in the neck regions of the loading side and opposite side implant regions (Fig. 4b). Stress fringes occurred on the second, third, and fourth implants for the IRPD with precision attachments. In general, as in all models, the lowest level of stress distribution was observed in the implant in the farthest region from the loading side (regions 16, 18, and 20) while high stress concentrations were observed in the implants on the loading side (regions 1, 3, 5, 6, and 10). Lower stress concentrations have been observed in the neck regions in a similar way with the clasp attachment (Fig. 4c, Fig. 5).

\section{Discussion}


Results indicate that the overdenture with locater attachments show minimal stress concentration compared with IRPD for the two implants models; however, in the locater attachments, fringes were observed only around the loaded side implants because of non-split attachment-type of implant design. For IRPD, bridge design with precious attachments demonstrate minimum stress compared with the crown design and clasp attachments-type designs for both the two and four implants models.

In many studies, stress patterns in the photoelastic model have been reported to be very similar to those of natural structures. ${ }^{15}$ This is because the stress distribution can be observed directly in the model and there is a similarity among the shape of stress, the area where it is formed, and natural structures. In photoelastic stress analysis models, cortical and cancellous bone are composed of a single resin. Therefore, even if the amount of stress changes, the areas where stress occurs will not; therefore, this method is useful for comparing stresses occurring in different prosthetic designs. ${ }^{16}$ Maximum biting force in individuals with natural teeth differ individually in different areas of the jaw. The largest biting force has been previously measured in premolar region at $415 \mathrm{~N}$ for females and $475 \mathrm{~N}$ for males. Average values of bite forces in premolar region for females $345 \mathrm{~N}$ and $401 \mathrm{~N}$ for males. ${ }^{17}$ MericskeStern and Zarb evaluated occlusal forces in a group of partial edentulous patients treated with an implant-supported fixed partial prosthesis and determined the maximum occlusal force to be $200 \mathrm{~N}$ for the first premolars and $300 \mathrm{~N}$ for the second premolars. ${ }^{18}$ In our study, a $300 \mathrm{~N}^{18,19}$ vertical force was applied to the right and left first premolars. The reason for the selection of this loading site being that food is chewed between premolars and molars in anterior loading applications. ${ }^{14}$ In a previous study, as a result of the application of laterotrusive load, a low level of stress was observed on the non-working side of the implant in the bar-ERA attachment, and the opposite arch interaction was determined to be low by providing balanced side contact. ${ }^{20}$ Furthermore, the highest stresses were formed in the posterior implants on the loading side..$^{20,21}$ In our study, similar to the results of previous studies, the highest stress value in all models occurred in the posterior implant on the loading side. In addition, similar to the results of Ochiai et al., ${ }^{12}$ the lowest stress level was observed in locator attachments where the implants were not splinted. In the study of Celik and Uludag, the fact that there were higher stress values in the locator attachments, in contrast to the present findings, might be due to the different retention forces of the retentive rubbers used. ${ }^{22}$ In our study, a blue-colored retentive rubber with a retention force of $1.5 \mathrm{lbs}=$ $66.7 \mathrm{~N}$ was used. This rubber was selected because the incoming forces are transmitted between the retentive rubber and the locator abutment, and a lower load is transferred to the implants. In this case, resilient design provides appropriate stress distribution characteristics. ${ }^{22}$

In addition, the fact that a lower stress concentration was observed around the implant in the model with locator attachment may be due to the location of the implant further away from the loading site in the locator attachment. The increased mucosa support may also be due to the fact that a portion of the force is transmitted to the alveolar crest on the loading point. ${ }^{23,24}$ While very low stress was observed in the implant on the non-loading side in the locator attachment, a moderate level was observed in the middle implants in the bar attachment and a low stress was observed in the opposing implants. This can be explained by the fact that the implants are connected to the bars and the stress is transferred to the other 
implants via the bar and the locator attachment, a resilience attachment preventing implant overloading ${ }^{25}$. However, the lack of stress distribution to the contralateral implant in the model with a locator attachment may be risky in terms of both bone loss monitoring in the implants on the loading side and, in particular, the deformation of locator attachment rubbers due to single side loading. Also in the present study, more stress fringes was observed in the overdenture prosthesis with bar attachment IO than the locator attachment. However, stress concentrations occurring not only on the loading side implants but also on the opposite side implants (1.22-1.08 N) indicated that the load was distributed equally to all implants by splinting. Fringes formed in this type of prosthesis with reduced tissue support were less sparse than those in the edentulous areas.

Hu et al evaluated the stress transfer of different overdenture attachment systems loaded directly over the implants, second premolar, and second molar regions. The authors stated that stress transfer to the edentulous crest region occurred and the stresses in the implants decreased as they approached the posterior area. At the same time, they observed that the difference between the stress transfer of overdenture attachment systems was also reduced as the load applied shifted to the posterior ${ }^{24}$. In our study, the low and moderate levels of stress values observed instead of high stress values, and the stress lines occurring in the region of the edentulous crest, might be due to the application of force from the premolar region. However, the stress lines observed in parts of the edentulous crest were greater in the two implant-supported models than in the four implant-supported models. This finding is comparable to those of a 10-year clinical follow-up by De Jong et al. in which the residual crest resorption of mandibular overdentures with two and four implants was compared. ${ }^{26}$ De Jong et al. concluded that a significantly greater atrophy was observed in the cases with two implants than in the cases with four implants. This result was observed to be independent of peri-implant bone loss, and this atrophy was believed to be formed due to the movement of the posterior prosthetic base towards the tissue under occlusal forces. ${ }^{26,27}$

There are many case reports in which researchers have used removable partial dentures combined with implant-supported fixed prostheses. ${ }^{6,8-11,27-29}$ Clinicians usually experience difficulty in providing effective and affordable implant restorations for patients with resorbed edentulous jaws. The resorbed residual alveolar crest and other anatomical limitations can prevent the placement of implants in the desired positions, especially in the posterior regions of the mandible. ${ }^{30,31}$ Implant placement in posterior areas of the jaws with anatomical limitations may be difficult or may require advanced surgical techniques. Despite these anatomical limitations, implant placement in the anterior regions of both jaws is generally reliable. ${ }^{30-32}$ The effective use of implants with a minimal number of removable dentures provides a more economical and comfortable treatment option for edentulous patients. ${ }^{31-33}$ Some of the total edentulous patients prefer implant-supported fixed restorations in the anterior region and removable partial dentures without requiring advanced surgical methods in the posterior regions while treatment options are being discussed. ${ }^{9}$ 
The present study was evaluated the different IRPD designs, also. This study is the first report evaluating the different type of IRPD designs. Accordin to our results, The stress concentration of crown-support and clasp attachment prosthesis was higher in the apical regions of the implants than in the cervical regions of the implants. However, when compared with other precious attachment models, the dentures with two implants with crown-support and clasp attachment generated higher stresses in the neck region, which can lead to marginal bone loss. It is thought that the use of precision attachments in IRPD prevents bone resorption in the implant and its surrounding. ${ }^{32-33}$

Bilhan et al evaluated the stress transfer of two-interforaminal implant-retained bridge and a removable partial denture with strain gauge method. ${ }^{25}$ They reported that, according to retentive anchors or bar attachments, the lowest strains occurred in the new design IRPD. This supports the results of our study. In the models with both two and four implants, the precision attachment of removable partial dentures generated less stress concentration then clasp attachment designs. Also splitting the anterior implants decreased the stress observation around the implants for this new treatment protocol. Crown design IRPD with clasp attachment was observed the highest stress values around the implants.

As mentioned in the case reports in the literature, the use of IRPD in total edentulous jaws enables more comfortable patient movement in terms of chewing, esthetics, and speech. $6,10,30$ When an overdenture is compared with full dentures, the pressure forces in the residual crest are also less common in 10 prostheses. ${ }^{33}$ At the same time, chewing movements become more balanced and regular in patients using IRPD. ${ }^{31-33}$ Bilhan et al also mentioned that the new design implant supported anterior bridge retained removable dentures with precission attachments eliminates two problems of conventional systems ease of hygiene with regard to bar attachment prosthesis and increased stability in severe resorbe mandible according to single attachments. ${ }^{25}$

As shown in a clinical study comparing removable partial dentures and conventional total prostheses; while the retention and comfort satisfaction of patients using removable partial dentures for mandibular prostheses was higher, a greater dissatisfaction in total prosthesis patients with mandibular prostheses was recorded. ${ }^{34}$ This suggests that the use of IRPD in total edentulous mandibular jaws is more appropriate. Collapse is observed in all physiological functions with old age; therefore, implant overdentures in elderly individuals should be designed to show satisfactory retention, be easily placed and removed, easy to clean, and cover a minimal surface area. In addition, it is important for younger total edentulous patients to feel comfortable in social settings with increased retention and to meet their esthetic expectations. ${ }^{27}$ IRPD and implant-supported fixed prosthetic restorations in the anterior region can have esthetic and psychologically positive effects so increase the self-confidence for young patients. In addition, prosthesis parts requiring less space for older patients can offer less complex retentive structures, and prosthetic components that will cause less plaque accumulation and are easy to maintain. Besides, in a recent study, wear of the matrices of unsplintted attachments are more remarkable especially the implants inserted not parallelly ${ }^{35}$, that cause more adjustment and maintenance in a short 
span of time. It was mentioned that splinting the angulated implants is an suitable solution and IRPD may also be a good alternative in this situtaions.

The present study is in vitro and presents limitations.

- in vivo studies and studies with other methodologies, such as finite element and strain gauge analysis, are necessary to complement the results found.

-stress transmission evaluated with static occlusal loads that limits the generalizability of our results. In fact, natural chewing is a dynamic movement and involves oblique forces. It would be beneficial for future studies to evaluate the stress transfer under different load directions.

-In this study, implant supported removable prosthetic designs were compared but for edentulous mandibles implant supported fixed prosthetic design such as hybrid prosthesis are commonly used in clinically. Therefore, for future studies both fixed and removable types can be investigated to extend the comparision.

\section{Conclusions}

Within the limitations of this study, it can be concluded that the IRPD prosthesis retained by an anterior bridge design with precision attachments is a suitable alternative treatment option. In particular, it can be used for patients who have anatomic inhibitions for full-mouth implant-supported fixed prosthesis, high esthetic expectations, denture adaptation difficulties, and inclined implants that block the retention of bar and locater attachments. For IRPD with precision attachment, the stress concentration was similar in two and four implant-supported removable dentures but was less than in clasp attachment designs.

\section{List Of Abbreviations}

- IO: Implant-supported overdentures

- IRPD: Implant-supported removable partial dentures

\section{Declarations}

Ethics approval and consent to participate: This study was approved by Selcuk University Faculty of Dentistry Noninvasive Clinical Research Ethical Committee [Approval number: 2011/01-04.01.2011].

Consent for publication: Not applicable. 
Availability of data and material: The datasets used and/or analyzed during the current study are available from the corresponding author on reasonable request.

Competing interests: The authors declare that they have no competing interests.

Funding: This study received financial support from Selcuk University Research Committee [grant\# 111020222] and also was supported by the Scientific Research Projects Coordination Unit, [grant\# 11102022]. The funders has no role in study design; in the collection, analysis and interpretation of data; in the writing of the report; and in the decision to submit the article for publication.

Authors' contributions: OI and GSO planned the study and consitited the hyposthesis. OI designed materialmethod and GSO collected and analyzed the datas. OI commented the datas. OYO and GSO researched the literature and writed the manuscript.

Acknowledgements: None

Authors' information: Gulsum Sayin Ozel; DDS PhD, Assist. Prof. Dr. Istanbul Medipol

Ozgur Inan; DDS PhD, Prof. Dr. Selcuk University, Faculty of Dentistry, Department of Prosthodontics, Konya, TURKEY

Ozgun Yusuf Ozyilmaz; Bezmialem University, Faculty of Dentistry, Department of Prosthodontics, Istanbul, TURKEY

\section{References}

1. Thomason JM, Kelly SAM, Bendkowski A, Ellis JS. Two implant retained over- dentures-a review of the literature supporting the McGill and York consensus statements. J Dent 2012;40:22-34.

2. Asami M, Kanazawa M, Lam TV, Thu KM, Sato D, Minakuchi S. Preliminary study of clinical outcomes for single implant-retained mandibular overdentures. J of Oral Sci. 2020;62(1):98-102.

3. Bryant SR, Walton JN, Macentee MI. A 5-year randomized trial to compare 1 or 2 implants for implant overdentures. J Dent Res 2015;94, 36-43.

4. Huang JZ, Lin XF. Evaluation of the clinical efficacy of implant-supported overdenture with edentulous mandible in elderly patients. West China j of stomatolog,2019;37(4):428-432. 
5. Ozyilmaz OY, Aykent F, Sayin Ozel G. Effect of mucosa thicknesses on stress distribution of implantsupported overdentures under unilateral loading: Photoelastic analysis.J Appl Biomater Func Mater.2019; 17(4), 2280800019882645.

6. Krennmair G, Seemann R, Fazekas A, Ewers R, Piehslinger E. Patient preference and satisfaction with implant-supported mandibular overdentures retained with ball or locator attachments: a crossover clinical trial.Int J Oral Maxillofac Implants. 2012; 27(6):1560-1568.

7. Zavanelli RA, Mazaro JVQ, Guilherme AS, Magalhães JB, Cardoso LC, Zavanelli AC. Distal extension removable partial denture with resilient attachment connected to an anterior fixed ımplant-supported prosthesis: a clinical report. World J Dent. 2012;3:87-90.

8. Uludag B. Hassas Tutucular İmplant vakalarında sorunlar ve çözümler implant destekli overdenture uygulamaları ve tutucu sistemler. Istanbul: Istanbul Ada Ofset; 2012. p. 175-7.

9. Feinberg E. Precision attachment case restoration with implant abutments: a review with case reports. J Oral Implantol 2011;37:489-98.

10. Uludag B, Celik G, Sahin V, Ozturk O. Fabrication of implant-assisted restorations utilizing functional impression techniques: case reports. J Oral Implantol. 2007;33:297-304.

11. Chronopoulos V, Sarafianou A, Kourtıs S. The use of dental implants in combination with removable partial dentures: a case report. J Esthet Restor Dent. 2008;20:355-65.

12. Ochiai KT, Williams BH, Hojo S, Nishimura R, Caputo AA. Photoelastic analysis of the effect of palatal support on various implant-supported overdenture designs. J Prosthet Dent. 2004;91:421-7.

13. Cehreli M, Duyck J, De Cooman M, Puers R, Naert I. Implant design and interface force transfer: a photoelastic and strain-gauge analysis. Clin Oral Implants Res. 2004;15:249-57.

14. Lee Jl, Lee Y, Kim YL, Cho HW. Effect of implant number and distribution on load transfer in implantsupported partial fixed dental prostheses for the anterior maxilla: a photoelastic stress analysis study. J Prosthet Dent.2016;115:161-9.

15. Teixeira FM, de Assis Claro CA, Neves AC, de Mello Rode S, da Silva-Concílio LR. Influence of loading and use of occlusal splint in implant-supported fixed prostheses. J Craniofac Surg. 2012;23:e477-80.

16. Machado AC, Cardoso L, Brandt WC, Henriques GE, de Arruda Nóbilo MA. Photoelastic analysis of the distribution of stress in different systems of overdentures on osseous-integrated implants. $J$ Craniofac Surg.2011;22:2332-6.

17. Padma S, Umesh S, Asokan S, Srinivas T. Bite force measurement based on fiber Bragg grating sensor.Journal of biomedical optics, 2017;22(10):107002.

18. Mericske-Stern R, Zarb GA. In vivo measurements of some functional aspects with mandibular fixed prostheses supported by implants. Clin Oral Implants Res. 1996;7:153-61.

19. Şahin S, Çehreli MC, Yalçın E. The influence of functional forces on the biomechanics of implantsupported prosthesis-a review. J Dent. 2002;30:271-82.

20. Çelik G, Uludağ B. Investigation by the photoelastic stress analysis of the effect of implant inclination and precision connections load transfer in the mandibular implant-retained overdenture 
designs. Turkiye Klinikleri J Dental Sci. 2013;19:17-25.

21. Zhang SJ, Zeng JY, Li J, Zhang R, Yin JY, Wang H. Photoelastic analysis of the influence of prosthetic material on the stress distribution in bone around implant. Chinese $\mathrm{j}$ of stomatolog, 2018; 53(1), 30-35.

22. Celik G, UludaG B. Photoelastic stress analysis of various retention mechanisms on 3-implantretained mandibular overdentures. J Prosthet Dent. 2007;97:229-35.

23. 23. Bilhan $\mathrm{H}$. The role of the dental implant in removable partial dentures. In: Şakar $\mathrm{O}$, editor. Removable partial dentures: a practitioners' manual.Cham: Springer International Publishing; 2016. pp. 195-208.

24. Hu F, Gong Y, Bian Z, Zhang X, Xu B, Zhang J, Shi X, Yu Y, Song L. Comparison of Three Different Types of Two-Implant-Supported Magnetic Attachments on the Stress Distribution in Edentulous Mandible. Comput Math Methods Med. 2019;2019:6839517. https://doi.org/10.1155/2019/6839517

25. Arat Bilhan S, Geckili O, Cilingir A, Bozdag E, Bilhan H.Evaluation of two interforaminal implants and implant-assisted removable dentures on stress distribution: an in vitro study. J Korean Assocof Oral Maxillofac Surg,2019; 45(4): 199-206.

26. de Jong MH, Wright PS, Meijer HJ, Tymstra N. Posterior mandibular residual ridge resorption in patients with overdentures supported by two or four endosseous implants in a 10-year prospective comparative study. Int J Oral Maxillofac Implants. 2010;25:1168-74.

27. Müller F, Schimmer M. Implant-supported overdenture prosthesis for edentulous elderly patients. Forum Implantol. 2013;2:97-103.

28. Pellecchia M, Pellecchia R, Emtiaz S. Distal extension mandibular removable partial denture connected to an anterior fixed implant-supported prosthesis: a clin.ççi cal report. J Prosthet Dent. 2000;83:607-12.

29. Starr NL. The distal extension case: an alternative restorative design for implant prosthetics. Int $J$ Periodontics Restorative Dent. 2001;21:61-7.

30. Mijiritsky E. Implants in conjunction with removable partial dentures: a literature review. Implant Dent. 2007;16:146-54.

31. Bassetti RG, Bassetti MA, Kuttenberger J. Implant-assisted removable partial denture prostheses: a critical review of selected literature. Int J Prosthodont. 2018;31:287-302.

32. Wismeijer D, Tawse-Smith A, Payne AG. Multicentre prospective evaluation of implant-assisted mandibular bilateral distal extension removable partial dentures: patient satisfaction. Clin Oral Implants Res. 2013;24:20-27.

33. Payne AG, Tawse-Smith A, Wismeijer D, De Silva RK, Ma S. Multicentre prospective evaluation of implant-assisted mandibular removable partial dentures: surgical and prosthodontic outcomes. Clin Oral Implants Res. 2017;28:116-125.

34. Celebić A, Knezović-Zlatarić D. A comparison of patient's satisfaction between complete and partial removable denture wearers. J Dent. 2003;31:445-51. 
35. Ortegón SM, Thompson GA, Agar JR, Taylor TD, Perdikis D. Retention forces of spherical attachments as a function of implant and matrix angulation in mandibular overdentures: an in vitro study. J Prosthet Dent 2009;101:231-8.

\section{Table}

Due to technical limitations, table 1 is only available as a download in the supplemental files section.

\section{Figures}

\section{Figure 1}

Photoelastic models of prostheses. a, Two implant-supported overdentures (IO) - locator; b, two implantsupported removable partial dentures (IRPD) bridge+clasp; c, two IRPD bridge+precision; d, two IRPD crown+clasp; e, four IO - bar; f, four IRPD bridge+clasp; and g, four IRPD bridge+precision.

政

\section{Figure 2}

Stress fringes of dentures with two implants. a, Stress fringes of dentures with two implants with locator attachment. b, Stress fringes of removable partial dentures over implant with two bridge-supported clasp attachment implants. c, Stress fringes of removable partial dentures over implant with two bridgesupported precision attachment implants. $d$, Stress fringes of removable partial denture over implant with two crown-supported clasp attachment implants. 
- marginal region $\quad$ apical region

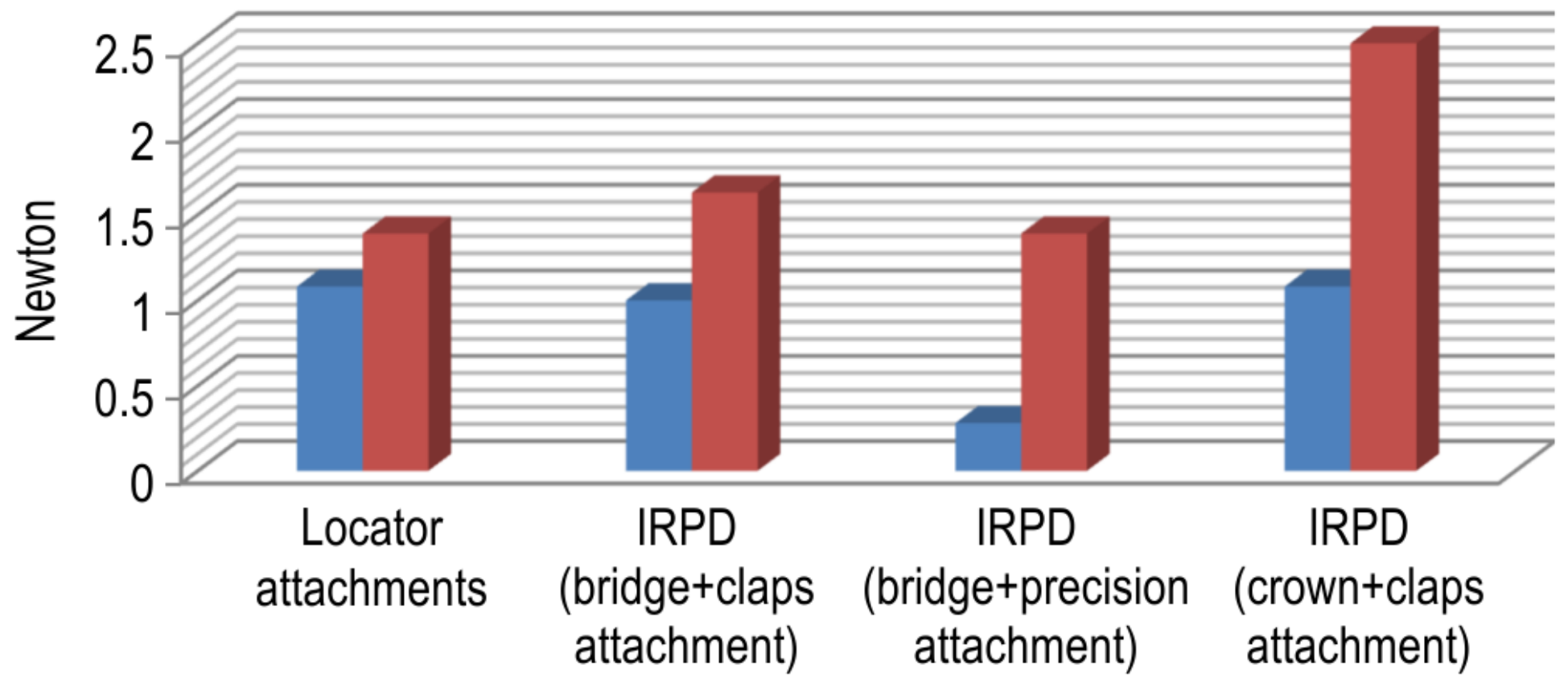

b

- marginal region $\quad$ apical region

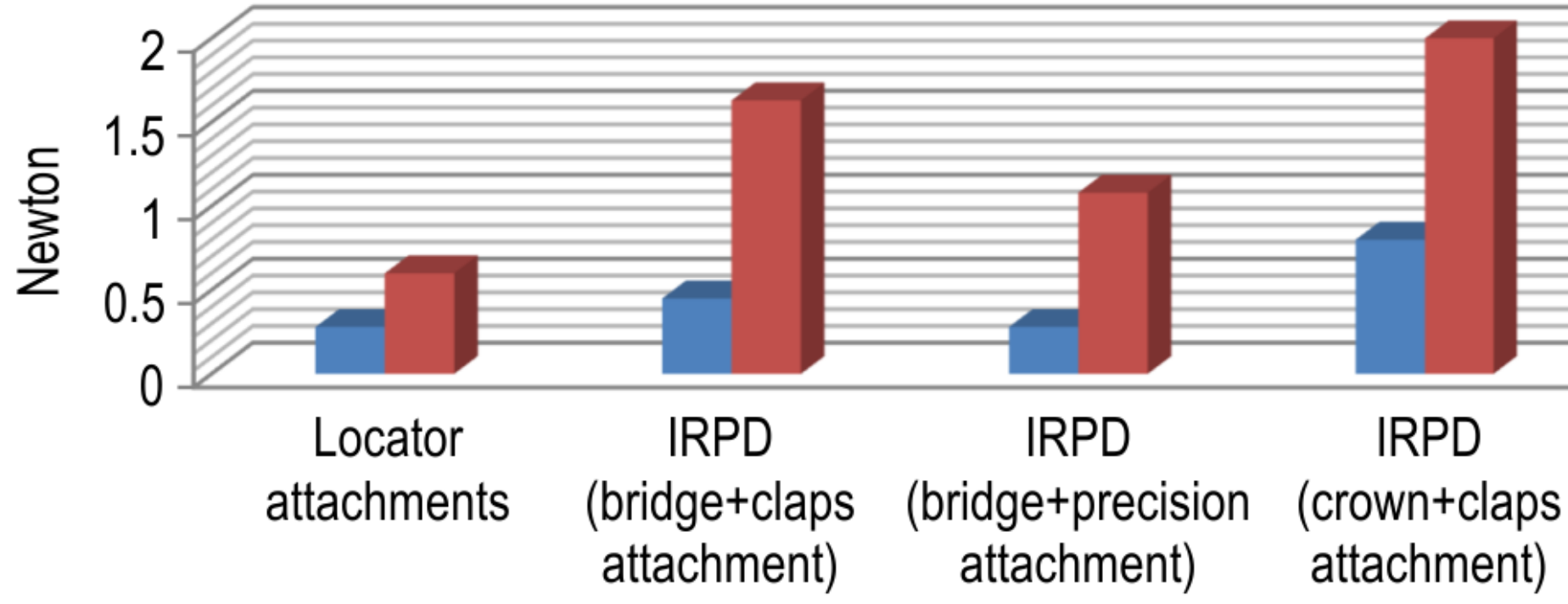

Figure 3

Stress fringe orders around loading sides of implants for dentures with two implants. a, Fringe orders around loading sides of ipsilateral implants for two implant-supported prosthesis: apical and marginal regions. b, Fringe orders around loading sides of contralateral implants for two implant-supported prostheses: apical and marginal regions. IRPD, implant-supported removable partial dentures. A

Figure 4 
Stress fringes of dentures with four implants. a, Stress fringes of overdenture total prosthesis with four implants and bar attachment. b, Stress fringes of removable partial denture over implant with four implants and bridge-supported clasp attachment. c, Stress fringes of removable partial denture over implant with four implants and bridge-supported precision attachment.

a

- marginal region a apical region

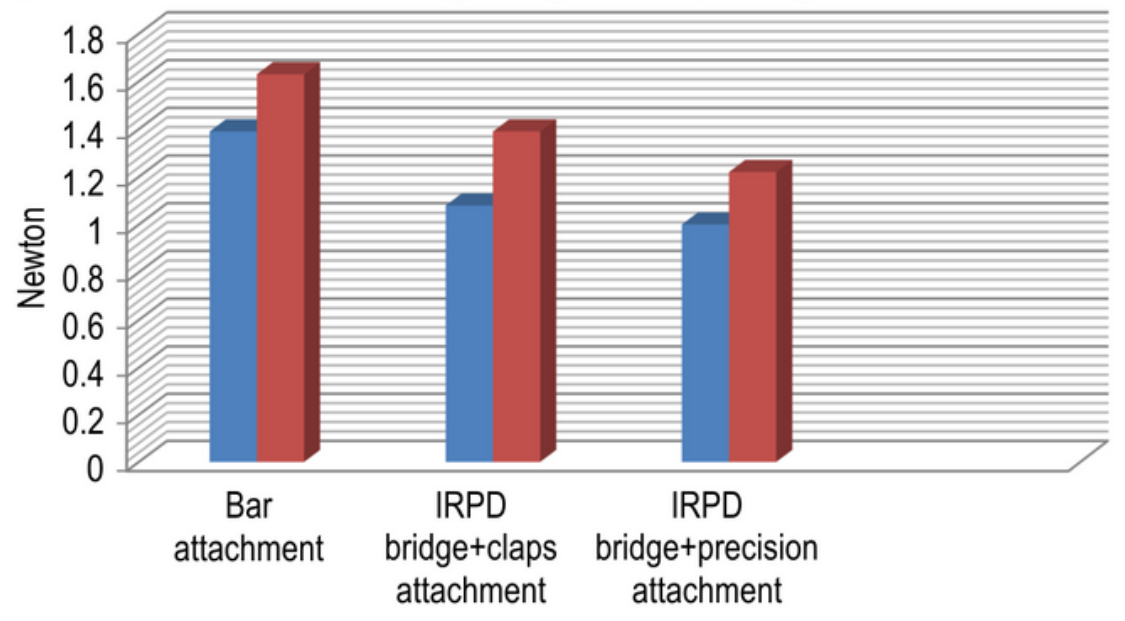

b $\quad$ marginal region $₫$ apical region

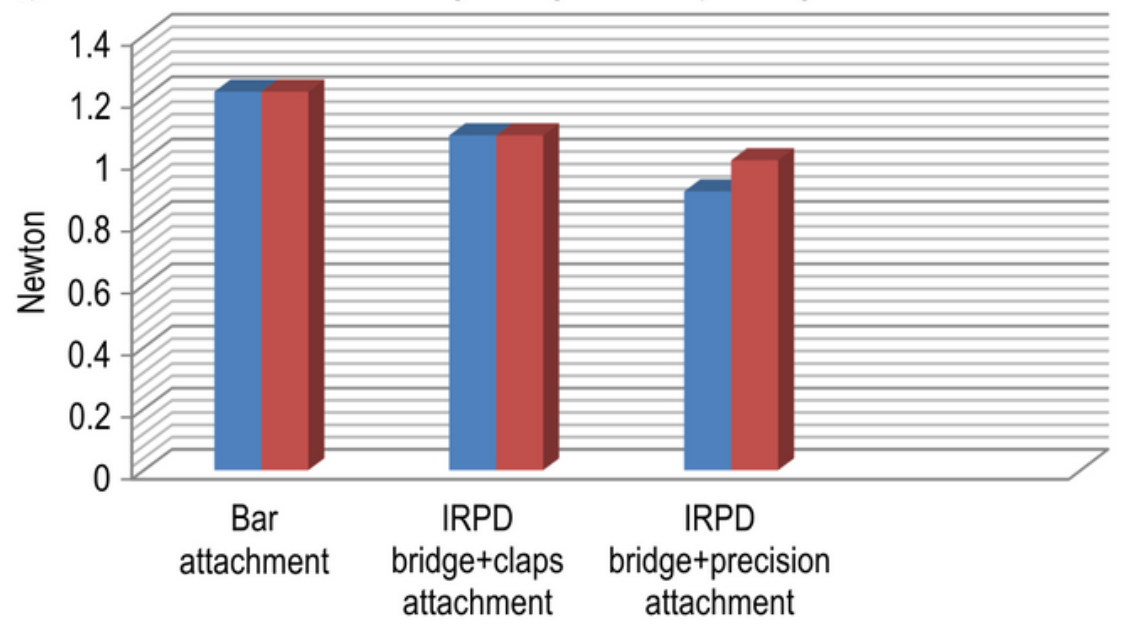

C $\quad$ marginal region $=$ apical region

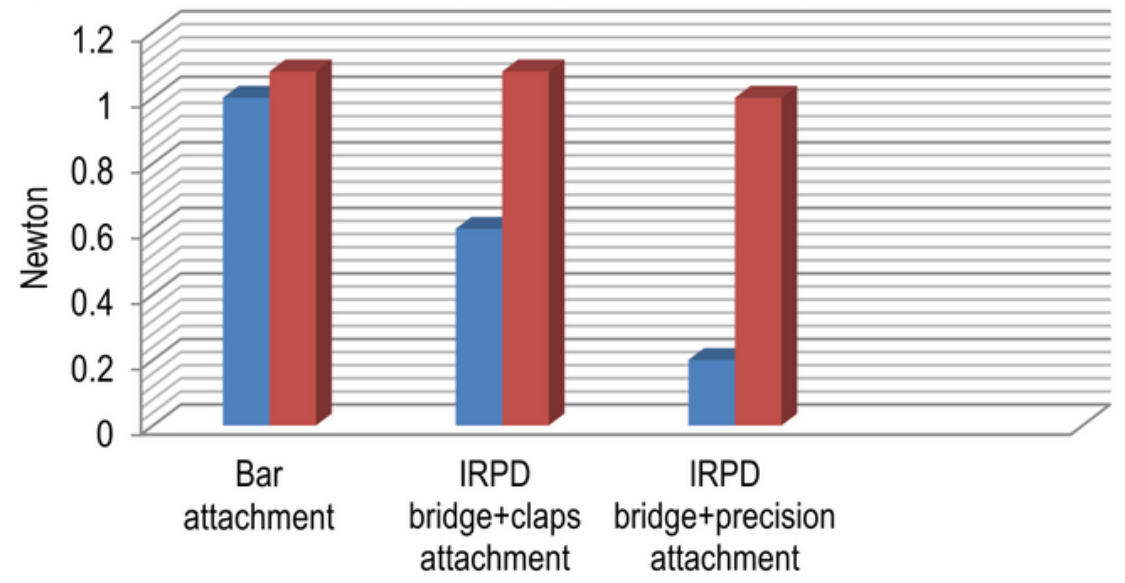

Figure 5 
Stress fringe orders around implants for dentures with four implants. a, Fringe orders around loading side of ipsilateral implants for four implant-supported prostheses: apical and marginal regions. b, Fringe orders around median implants for four implant-supported prosthesis: apical and marginal regions. c, Fringe orders around loading side of contralateral implants for four implant-supported prosthesis: apical and marginal regions. IRPD, implant-supported removable partial dentures.

\section{Supplementary Files}

This is a list of supplementary files associated with this preprint. Click to download.

- Table1.docx 